\title{
Hepatic Vein-Sparing Hepatectomy for Multiple Colorectal Liver Metastases at the Caval Confluence
}

\author{
Guido Torzilli, MD, PhD, FACS, Fabio Procopio, MD, Matteo Cimino, MD, Matteo Donadon, MD, \\ Daniele Del Fabbro, MD, Guido Costa, MD, and Carlos A. Garcia-Etienne, MD
}

Department of Hepatobiliary and General Surgery, Humanitas Research Hospital, University of Milan-School of Medicine, Rozzano, MI, Italy

\begin{abstract}
Background. Two-stage hepatectomies generally are selected for patients with multiple bilobar colorectal liver metastases (CLMs) involving the hepatic veins (HV) at the caval confluence to reduce the risk of postoperative hepatic failure due to insufficient remnant liver. ${ }^{1,2}$ The use of IOUS based on well-established criteria offers alternative technical solutions to the staged resections. ${ }^{3,4}$ This report describes a sophisticated IOUS-guided parenchyma-sparing procedure.

Methods. A 57-year-old woman with multiple CLMs underwent surgery. One of these CLMs was located in segments 8 to 4 sup involving the middle hepatic vein (MHV) at the caval confluence. A second CLM was between dorsal segment 8 and the paracaval portion of segment 1 involving the right hepatic vein (RHV) at the caval confluence. Neither the inferior RHV nor the communicating veins were evident at preoperative imaging. The left hemiliver represented $27 \%$ of the total liver volume, and segments 2 and 3 represented $16 \%$.

Results. After a J-shaped thoracophrenolaparotomy, liver exploration with IOUS showed tumoral invasion of MHV and RHV at their caval confluence for one third of their circumference. No communicating veins were intraoperatively evident. A partial resection of segments 7, 8, and 4 superior and 1-paracaval sparing both RHV and MHV was
\end{abstract}

Electronic supplementary material The online version of this article (doi:10.1245/s10434-014-4189-1) contains supplementary material, which is available to authorized users.

(C) Society of Surgical Oncology 2014

First Received: 25 April 2014;

Published Online: 29 October 2014

G. Torzilli, MD, PhD, FACS

e-mail: guido.torzilli@ humanitas.it performed. The latter were partially resected, and vessel wall reconstruction was obtained by direct running suture. No congested area or vascular thrombosis occurred, and the postoperative course was uneventful. No local recurrence had occurred after 6 months of follow-up evaluation.

Conclusions. The video shows an HV-sparing IOUS-guided hepatectomy as an alternative to conventional staged surgery. This policy represents a safe and effective alternative to major resection performed immediately or in a staged perspective.

\section{REFERENCES}

1. Schnitzbauer AA, Lang SA, Goessmann H, et al. Right portal vein ligation combined with in situ splitting induces rapid left lateral liver lobe hypertrophy enabling 2-staged extended right hepatic resection in small-for-size settings. Ann Surg. 2012;255:405-14.

2. Jaeck D, Oussoultzoglou E, Rosso E, et al. A two-stage hepatectomy procedure combined with portal vein embolization to achieve curative resection for initially unresectable multiple and bilobar colorectal liver metastases. Ann Surg. 2004;240:1037-49.

3. Torzilli G, Palmisano A, Procopio F, et al. A new systematic small for size resection for liver tumors invading the middle hepatic vein at its caval confluence: mini-mesohepatectomy. Ann Surg. 2010; 251:33-9.

4. Torzilli G, Donadon M, Marconi M, et al. Systematic extended right posterior sectionectomy: a safe and effective alternative to right hepatectomy. Ann Surg. 2008;247:603-11. 